\title{
Light bullets formation accompanied by odd harmonics genera- tion under Mid-IR femtosecond filamentation in LiF
}

\author{
Evgeniya Smetanina ${ }^{1, \star}$ \\ ${ }^{1}$ CELIA (Centre Lasers Intenses et Applications) Université Bordeaux Domaine du Haut-Carré 43 rue Pierre \\ Noailles 33405 Talence Cedex France
}

\begin{abstract}
The formation of light bullets in mid-IR femtosecond filamentation is investigated numerically by means of the unidirectional pulse propagation equation (UPPE). A general approach for light bullets formation is presented for condensed media under anomalous GVD. The main parameters of the light bullets are evaluated. The features of super continuum and odd harmonic generation accompanying the light bullets formation are analyzed.
\end{abstract}

An appearance of new hight-power femtosecond laser sources in MID-IR wavelength region [1] leads to an active research of MID-IR laser filamentation regimes in both, condensed transparent dielectrics [2,3] and gases [4-6]. The wavelengths from 2.5 to $4 \mu \mathrm{m}$ correspond to the anomalous group velocity $(\mathrm{GVD})$ region in a large number if optical materials (silica glass, $\mathrm{CaF}_{2}, \mathrm{BaF}_{2}, \mathrm{LiF}$, YAG). Thus, an interplay between Kerr nonlinearity and anomalous GVD leads to a formation of spatially and temporally compressed wavepackets - light bullets (LB) [7-9]. In the current work we present the numerical results on the filamentation of $3.1 \mu \mathrm{m}$ femtosecond laser pulse in LiF. The formation of the LB and evolution of the pulse spectrum will be considered.

The modeling was performed by means of unidirectional pulse propagation equation. Two $\chi^{(3)}-$ based effects are included: Kerr nonlinearity and third harmonic generation, that leads to a cascade generation of odd harmonics. We consider a weakly focused linearly polarized 3.1- $\mu \mathrm{m}$ laser pulses with peak power $\mathrm{P}=1.5 P_{c r}$, where the critical power of the self-focusing is $P_{c r}$ equals to $100 \mathrm{MW}$.

We report, that the main spatio-temporal features of the LB formation at $3.1 \mu \mathrm{m}$ in $\mathrm{LiF}$ are similar to those for LBs formation at 1.8 $\mu \mathrm{m}$ in fused silica [8]. The duration of the LB reaches the value about 2-3 optical cycles (Fig.1 a-b). The odd harmonic generation accompanies the pulse propagation and the LB formation. The peak intensity of the harmonics oscillates along the propagation distance with the period corresponding to the phase matching conditions. The obtained results are close to the odd harmonic generation during filamentation in fused silica and $\mathrm{CaF}_{2}$ [3]. The closer the pulse is to the nonlinear focus, the wider are the harmonics spectra. Finally, the harmonics spectra are overlapped with the widely broadened blue wing of supercontinuum that appears due to the self-steepening of the pulse and corresponds to the LB formation (Fig. $1 \mathrm{c}$ ). The beating of the harmonics intensity along the propagation distance provides the beating of the pulse peak intensity in spatio-temporal domain (Fig.1 d). Together with that, the generated odd harmonics may lead to appearance of new ionization and color-centers formation pathways with lower number of photons.

^e-mail: evgeniya.smetanina@u-bordeaux.fr 
a)
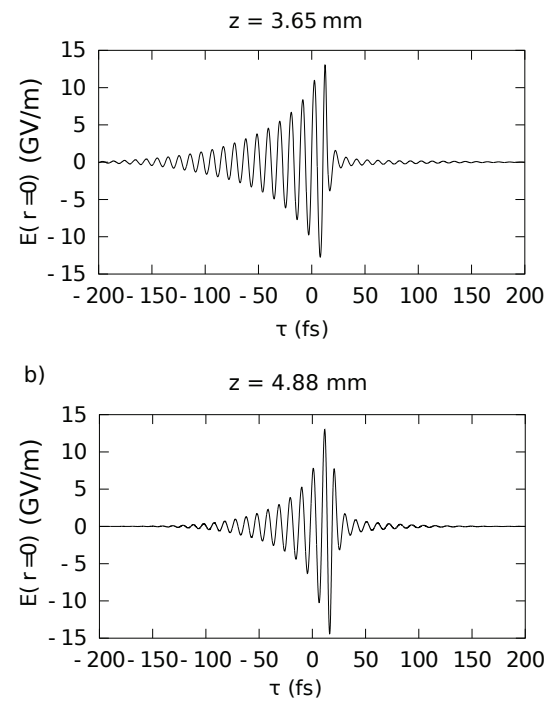

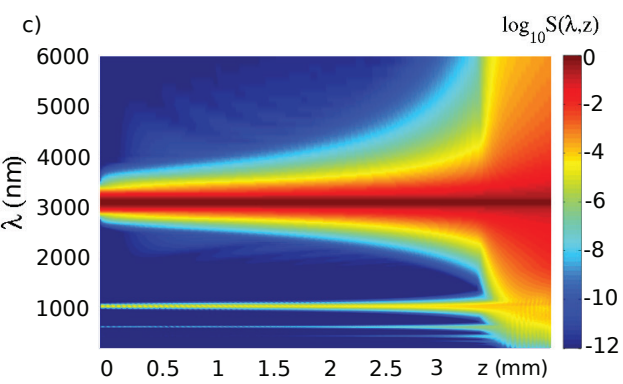

d)

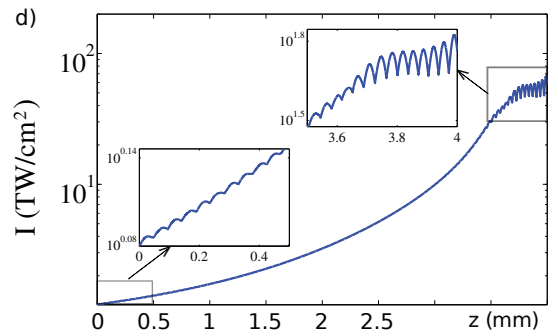

Figure 1. a)-b) LB time profiles of the electric field $E(\tau, r=0)$, the propagation distance $\mathrm{z}$ is specified on the figure, c) evolution of the angularly integrated spectrum $S(\lambda, z)$ along the laser pulse propagation axis $z$ under filamentation in LiF. $\tau<0$ corresponds to the pulse front. $\mathrm{S}(\lambda, z)$ is normalized on its maximum value at each $\mathrm{z}$. The incident pulse parameters are $\mathrm{a}_{0}=90 \mu \mathrm{m}, \tau_{0}=120 \mathrm{fs}$ in a) and $\tau_{0}=60 \mathrm{fs}$ in $\mathrm{b}$ ), the focal distance is $5 \mathrm{~mm}$.

Prof.V.P. Kandidov is acknowledged for fruitful discussions. Prof. S.Skupin is acknowledged for the opportunity to use the UPPE cod "CUPRAD". This work was granted access to the HPC resources of TGCC under the allocation A0010506129 made by GENCI.

\section{References}

[1] R. Butkus, R. Danielius, A. Dubietis, A. Piskarskas, A. Stabinis, Applied Physics B 79, 693 (2004)

[2] S.V. Chekalin, V.O. Kompanets, A.V. Kuznetsov, A.E. Dormidonov, V.P. Kandidov, Laser Physics Letters 13, 065401 (2016)

[3] N. Garejev, V. Jukna, G. Tamošauskas, M. Veličkè, R. Šuminas, A. Couairon, A. Dubietis, Opt. Express 24, 17060 (2016)

[4] S.V. Chekalin, A.E. Dokukina, A.E. Dormidonov, V.O. Kompanets, E.O. Smetanina, V.P. Kandidov, Journal of Physics B: Atomic, Molecular and Optical Physics 48, 094008 (2015)

[5] P. Panagiotopoulos, P. Whalen, M. Kolesik, J.V. Moloney, Nat Photon 9, 543 (2015)

[6] N.A. Panov, D.E. Shipilo, V.A. Andreeva, O.G. Kosareva, A.M. Saletsky, H. Xu, P. Polynkin, Phys. Rev. A 94, 041801 (2016)

[7] L. Bergé, S. Skupin, Phys. Rev. Lett. 100, 113902 (2008)

[8] E.O. Smetanina, V.O. Kompanets, A.E. Dormidonov, S.V. Chekalin, V.P. Kandidov, Laser Physics Letters 10, 105401 (2013)

[9] I. Gražulevičiūtè, G. Tamošauskas, V. Jukna, A. Couairon, D. Faccio, A. Dubietis, Opt. Express 22, 30613 (2014) 